\title{
Heart-lung interactions in COVID-19: prognostic impact and usefulness of bedside echocardiography for monitoring of the right ventricle involvement
}

\author{
Michael Dandel ${ }^{1,2}$ (D) \\ Accepted: 7 April 2021 / Published online: 17 April 2021 \\ (c) The Author(s), under exclusive licence to Springer Science+Business Media, LLC, part of Springer Nature 2021
}

\begin{abstract}
Due to the SARS-CoV-2 infection-related severe pulmonary tissue damages associated with a relative specific widespread thrombotic microangiopathy, the pathophysiologic role of heart-lung interactions becomes crucial for the development and progression of right ventricular (RV) dysfunction. The high resistance in the pulmonary circulation, as a result of small vessel thrombosis and hypoxemia, is the major cause of right heart failure associated with a particularly high mortality in severe COVID-19. Timely identification of patients at high risk for RV failure, optimization of mechanical ventilation to limit its adverse effects on RV preload and afterload, avoidance of medication-related increase in the pulmonary vascular resistance, and the use of extracorporeal membrane oxygenation in refractory respiratory failure with hemodynamic instability, before RV failure develops, can improve patient survival. Since it was confirmed that the right-sided heart is particularly involved in the clinical deterioration of patients with COVID-19 and pressure overload-induced RV dysfunction plays a key role for patient outcome, transthoracic echocardiography (TTE) received increasing attention. Limited TTE focused on the right heart appears highly useful in hospitalized COVID-19 patients and particularly beneficial for monitoring of critically ill patients. In addition to detection of right-sided heart dilation and RV dysfunction, it enables assessment of RV-pulmonary arterial coupling and evaluation of RV adaptability to pressure loading which facilitate useful prognostic statements to be made. The increased use of bedside TTE focused on the right heart could facilitate more personalized management and treatment of hospitalized patients and can contribute towards reducing the high mortality associated with SARS-CoV-2 infection.
\end{abstract}

Keywords COVID-19 $\cdot$ Echocardiography $\cdot$ Heart-lung interaction $\cdot$ Pulmonary vessel thrombosis $\cdot$ Right ventricle $\cdot$ Heart failure

\section{Introduction}

Coronavirus disease 2019 (COVID-19) is caused by the severe acute respiratory syndrome corona-virus 2 (SARS$\mathrm{CoV}-2$ ), a novel coronavirus related to the SARS-CoV and Middle East respiratory syndrome coronavirus (MERS-CoV) [1]. Compared with SARS and MERS, COVID-19 exerts more damages on the cardiovascular system, most notably life-threatening pulmonary vessel injury and cardiac dysfunction, with and without direct myocardial injury $[1,2]$.

Michael Dandel

mdandel@aol.com

1 German Centre for Heart and Circulatory Research (DZHK) Partner Site, Berlin, Berlin, Germany

2 Cardio Centrum, Berlin, Germany
The primary pulmonary injury and the subsequent cardiovascular complications constitute the key pathophysiology of COVID-19 [3]. Pulmonary vessel injuries with blood flow alterations, followed by right heart dilation and right ventricular (RV) failure, were found among the major causes of death related to COVID-19 [3-5]. In addition, critically ill COVID-19 patients are at particularly high risk of pulmonary embolism (PE) associated with massive RV pressure overload [6].

This article provides an overview on the clinical relevance of COVID-19-associated RV dysfunction and on the usefulness of right heart assessment by transthoracic echocardiography (TTE) in hospitalized patients. Special attention is focused on the benefits of bedside limited TTE in critically ill COVID-19 patients, where it is increasingly gaining importance for clinical decision-making. 


\section{Major COVID-related pulmonary and cardiovascular injuries}

Like other coronaviruses, SARS-CoV-2 mainly affects the lung tissue. In an early study, all hospitalized COVID-19 patients had pneumonia confirmed by chest CT. [7]. In large study, $48.8 \%$ of hospitalized patients had severe pneumonia requiring high-flow $\mathrm{O}_{2}$ therapy [8]. Adults admitted to emergency departments with suspected COVID-19 that were subsequently confirmed were more than twice as likely to die or receive organ support as those who did not have COVID-19 confirmed, despite having similar age and comorbidities (expect chronic lung disease) [9]. When compared to patients with pneumonia of other etiologies, those with COVID-19 appeared more likely to develop hypoxemic respiratory failure and acute respiratory distress syndrome (ARDS) which was also associated with higher mortality $[10,11]$. In a large study, $41 \%$ of COVID-19 patients necessitating intensive care developed ARDS [12]. COVID-19-related ARDS (CARDS) revealed particularly high mortality rates (between 54 and 85\%) [13-16]. However, for patients with ARDS of different other etiologies, a meta-analysis revealed for the years 2000-2015 an overall mortality rate of $45 \%$ [17]. The strikingly high mortality related to CARDS raises important questions regarding the reasons for this discrepancy. One of the most likely explanations for the high mortality could be the particularly high incidence and severity of cardiovascular complications associated with SARS-CoV-2 infections.

\section{Clinical and prognostic relevance of cardiovascular complications}

In addition to pulmonary damages, SARS-CoV-2 can cause both direct and indirect cardiovascular injuries, including acute corpulmonale and cardiogenic shock, thrombotic complications (including PE), acute coronary syndromes, myocardial injury, and severe arrhythmias [2, 18, 19]. Microthrombosis in various organs, which is the most prominent clinical feature of COVID-19, was demonstrable in $305(91.3 \%)$ of 334 autopsies [20]. Autopsies revealed a major contribution of pulmonary vessel damages to COVID-19-related deaths [21-23]. Pulmonary small vessel thrombi and platelet agglutination are distinctive features of COVID-19 being also related to its poor prognosis $[19,22]$. Between 80 and $100 \%$ of the lungs examined at autopsy revealed platelet-fibrin thrombi in the small pulmonary arterial and venous vasculature [22, 24, 25]. Histological analyses of the pulmonary vessels of deceased SARSCoV-2-infected patients found that microangiopathy with widespread thrombosis (including alveolar capillary microthrombi) were 9 times more frequent than in the lungs from patients who died from influenza [23]. One study revealed microthrombosis within small pulmonary arteries of all persons who died from COVID-19, and in all cases the cause of death was found within the lungs or the pulmonary vascular system [26]. The incidence of PE in COVID-19 patients is also particularly high (around 7\% in hospitalized patients and up to one-third of those requiring intensive care unit) and is associated with a significantly higher mortality risk compared to COVID-19 patients without PE [27, 28].

Simultaneous occurrence of ARDS and high D-dimer plasma levels in COVID-19 appeared associated with high mortality. In two studies, all deceased patients with COVID19 had high D-dimer values [25, 29]. D-dimer values were found up to 14 times higher in deceased patients with COVID-19 than in survivors with moderate COVID-19 [29]. In patients with high D-dimers and low compliance of the respiratory system, the 28-day mortality was found 4.3 times higher compared to those with low D-dimers and high compliance (56\% and $13 \%$, respectively) [30]. CT also revealed filling defects or occlusions of the pulmonary vasculature that were more prominent in patients with high D-dimer levels ( $94 \%$ of them had bilateral, diffuse areas of hypoperfusion, consistent with the presence of thrombi or emboli) indicating that pulmonary vascular thrombosis could be the main cause of COVID-19-related death [30]. Pulmonary vascular thrombosis and the relatively high incidence of $\mathrm{PE}$ can also explain the high prevalence of pulmonary hypertension $(\mathrm{PH})$ with dilated RV and relatively small left ventricular (LV) in symptomatic COVID-19, as well as the autopsy findings were RV dilation with concurrently reduced LV diameters was the most significant macroscopic cardiac abnormality [19, 22, 31]. In one study, RV dilation with RV:LV diameter ratios $>1.1$ was found at autopsy in $41 \%$ of patients who died of COVID-19 [28]. In hospitalized patients, RV dilation and systolic pulmonary arterial pressure (PAPs) appeared significantly correlated with D-dimer levels [32].

Myocardial injuries, revealed by elevated cardiac biomarkers, were detected in up to $30 \%$ of hospitalized patients with COVID-19, and in those with preexisting cardiovascular diseases, the prevalence of elevated biomarkers reached 55\% [2]. The contribution of cardiac dysfunction to the mortality of COVID-19 patients, either alone or in conjunction with respiratory failure, can reach $43 \%$ [33]. Inhospital COVID-related death was found twice as high in patients with HF history than in those without [34]. In one study, $20 \%$ of the hospitalized COVID-19 patients showed increased high-sensitive troponin I (hs-TnI) blood levels with or without additional abnormalities in electrocardiography (ECG) or echocardiography (ECHO) and in these patients the mortality reached $51 \%$, whereas in those with normal hs-TnT, the mortality rate was only $4.5 \%$ [33]. Increased hs-TnI was identified as an independent risk factor for COVID-19-related mortality [35, 36]. In a multicenter study, $62 \%$ of the hospitalized COVID-19 patients had 
bio-marker evidence of myocardial injury (high troponin), but myocardial injury alone was not a predictor of increased mortality [13]. In those with high troponin accompanied by ECHO abnormalities, the inhospital mortality reached $31.2 \%$, and high troponin in combination with ECHO evidence of RV dysfunction was associated with a threefold increased risk of death [13]. In another study, the mortality rate for patients without any underlying cardiovascular disease (CVD) and normal TnT levels was $13 \%$, whereas in patients with an underlying CVD plus elevated TnT, the mortality reached $69.4 \%$ [37]. A prospective study also revealed hs-TnI as the most relevant predictive factor for ICU referral in COVID-19 patients and hs-TnI appeared significantly correlated with SPAP values and TAPSE [32]. Given that in PE not related to COVID-19, the combined effect of $\mathrm{RV}$ afterload mismatch and myocardial ischemia can lead to progressive RV dysfunction associated with elevated TnT [38]; the high troponin levels and their prognostic value in severe COVID-19 could also be explained by the deleterious impact of the pulmonary microthrombosis-induced RV myocyte damage related to the high wall stress caused by acute overload of the RV [32]. The detection of RV dysfunction in $50 \%$ of the patients with COVID-19 associated with elevated TnI, but normal LV function, supports this assumption [39]. In a pediatric study, the RV free wall longitudinal strain was the strongest predictor of myocardial injury defined by elevated brain natriuretic peptide (BNP) and/or TnI levels [40].

Acute heart failure (HF) can be the primary presenting manifestation of COVID-19 [14, 18]. It was present in 23\% of patients with COVID-19 at their initial presentation and in $52 \%$ of those who finally died in the ICU [14]. There is evidence that pulmonary microvascular damages and occlusion by neutronphil aggregates and platelet-fibrin thrombi responsible for the particularly severe acute respiratory failure may have also an important contribution to the development of COVID-19-related acute HF [21].

Given the high risk of life-threatening acute cardiorespiratory failure triggered by the extensive pulmonary vessel thrombosis, clinical deterioration of the respiratory function or the lack of improvement despite of supportive therapy, together with laboratory evidence of severe inflammation and hypercoagulable state, as well as the appearance of the first ECHO and/or ECG signs of RV pressure overload should prompt rapid diagnostic procedures to identify patients with pulmonary in situ microthrombosis or PE [41]. In this regard, particularly noteworthy is the detection of ECG signs suggesting acute RV pressure overload in 30\% of hospitalized patients with COVID-19 [42]. This indicates that ECG could be particularly useful for rapid identification of patients at higher risk for RV failure already at the time of admission to the hospital and close monitoring of hospitalized patients by ECG could also be useful for the timing of ECHO examinations.
LV dysfunction appeared not to be a major cause for COVID-19-associated HF [16, 19, 22]. One study found no differences in the incidence of congestive HF between COVID-19 and non-COVID-19 patients with ARDS [11]. An ECHO study revealed no differences in the LV ejection fraction (LVEF) between patients treated in the ICU and those who did not need intensive care [19]. In another study, most patients with elevated troponin had preserved LV function [13]. In an autopsy study where thrombotic microangiopathy was identified in all lungs, the coronary arteries showed no significant stenoses or acute thrombus formation [22].

Although acute myocarditis is a recognized severe complication of the disease, the initially presumed relevant contribution of to the morbidity and mortality of COVID-19 is still debated. In a large recent multi-center study, acute myocarditis was confirmed only in $<1 \%$ of patients with COVID-19 [43]. An autopsy study showed no evidence of myocarditis and the lack of relevant myocardial necrosis which could impair myocardial pump function explains the relatively few cases of COVID-19-associated severe LV dysfunction [22].

\section{Pathophysiology of cardiovascular involvement in COVID-19}

Similar to SARS-CoV, SARS-CoV-2 also uses the angiotensin-converting enzyme 2 (ACE2) receptor to invade pulmonary alveolar cells and other intra- or extra-pulmonary cells with ACE2 receptors [2, 44]. The higher affinity of binding to the ACE2 receptor revealed by SARS-CoV-2 may contribute to the increased transmissibility of COVID-19 and the usually higher degree infection severity [44]. ACE2 receptors are highly expressed in cardiovascular tissue, and SARS-CoV-2 may spread via the bloodstream and produce direct viral injury [2]. However, the pathophysiology underlying cardiac involvement during SARS-CoV-2 infection is likely to be multifactorial (direct viral toxicity, cytokinemediated endothelial damage and thrombotic events, overloading-induced RV dysfunction) [2].

The pathophysiologic role of the heart-lung interactions appeared mainly involved in the development and progression of severe RV dysfunction (Table 1; Fig. 1), which in turn has a major contribution to the poor prognosis of CARDS. Due to the particularly distinctive hyper-inflammatory and pro-thrombotic state, extensive pulmonary in situ microthrombosis and $\mathrm{PE}$ rather than pneumonia appeared to be more often not only the major cause of respiratory failure but also the major cause of acute decompensated HF, where the excessively increased RV afterload has a crucial pathogenic role. Acute RV failure is a serious clinical challenge regardless of its etiology because it complicates the use of recommended treatment strategies in critically ill patients 
Table 1 Heart-lung interactions in patients with COVID-19

Studies $\quad$ Relevance of heart-lung interactions in COVID-19

Fox et al. $[22,31]$

Dolhnikoff et al. [24]

García-Cruz et al. [59]

Zeng et al. [19]

Mahmoud-Elsayed et al. [60] • The chief ECHO abnormalities in 78 investigated patients were RV dilatation and RV dysfunction in $41 \%$ and $27 \%$, respectively. RV impairment was associated with increased D-dimer levels

- LV function was hyperdynamic or normal in most (89\%) patients

Goudot et al. [32]

- In hospitalized patients (56\% of them in the ICU) hs-TnI and D-dimer levels were significantly correlated with both PAPs and TAPSE. Additionally, D-dimer levels correlated also with RV dilation $(p=0.01)$

- The high predictive value of elevated hs-TnI for ICU referral in patients without LV alterations, together with the correlation of D-dimer with RV dilatation, confirms the relevance of COVID-19-associated pulmonary microvascucular thrombosis-induced RV afterload increase with the resulting high wall tension-induced RV myocardial damage and dysfunction

Giustino et al. [13]

- $26 \%$ of patients with biomarker evidence of myocardial injury had ECHO signs of RV dysfunction which was associated with a three-fold increased risk of death

Argulian et al.[61]

- ECHO revealed RV dilation in $31 \%$ of the hospitalized patients with similar prevalence of major co-morbidities, laboratory markers of myocardial injury or inflammation, and also similar LV size and EF compared with those without RV dilation

- Nearly 4 times higher mortality rate in patients with RV dilation than in those without dilation (41\% and $11 \%$, respectively). On multivariate analysis, RV enlargement was the only variable significantly associated with mortality

Li et al. [62]

- ECHO monitoring reveled that compared with survivors, non-survivors displayed enlarged right heart chambers, reduced RV function and elevated PAPs

- RVLS, $\mathrm{FAC}_{\mathrm{RV}}$, and TAPSE were associated with higher mortality (area under the curve 0.87, 0.72 and 0.67, respectively)

D’Alto et al. [16] - COVID 19-induced ARDS is associated with early RV-PA uncoupling. 25 (67\%) of the 37 invasively ventilated patients died. Non-survivors had nearly 3 times higher D-dimer plasma levels $(\mathrm{p}<0.001)$ as well as higher PAPS and lower TAPSE $(\mathrm{p}<0.001)$. Reduction of TAPSE/PASP was identified as an independent predictor of mortality. In non-survivors LV size was normal, and LVEF was $\geq 50 \%$

Szekely et al. [39]

- RV dilation and dysfunction were the most frequent ECHO abnormalities (detectable in $39 \%$ of patients) with a 5 times higher incidence than the reduction of LVEF. RV dysfunction correlated with troponin levels. $\bullet$ During follow-up, $50 \%$ of patients showing clinical deterioration revealed also further worsening of RV parameters, whereas the LV function remains normal in all those patients

Schott et al. [63]

- ECHO revealed RV dilation and increased RV/LV ratio in the majority of hospitalized patients (74\% and $82 \%$, respectively), whereas reduction of LVEF was detected only in $3 \%$ of the patients

Rivinius et al. [64]

- In mechanically ventilated heart transplant recipients with COVID-19, the mortality of can reach $87 \%$ and appeared associated with RV dysfunction accompanied by increased PAP, whereas none of them showed LVEF reduction

Caravita et al. [65]

- The prevalence of PH (mean PAP $\geq 25 \mathrm{mmHg}$ ) was 4 times higher in ventilated COVID-19 patients with ARDS compared to those without the need for mechanical ventilation $(76 \%$ vs. $19 \%, \mathrm{p}<0.001)$

Doyen et al. [66] (47\% and $13 \%$, respectively)

$R V$ right ventricle, $I C U$ intensive care unit, $E C H O$ echocardiography, $P A P s$ systolic pulmonary arterial pressure, $L V$ left ventricle, $E F$ ejection fraction, $R V L S$ right ventricular longitudinal strain, $F A C_{R V}$ right ventricular fractional area change, TAPSE tricuspid annulus peak systolic excursion, $P H$ pulmonary hypertension, $P A P$ pulmonary arterial pressure, $A R D S$ acute respiratory distress syndrome, $h s$-TnI high-sensitive troponin I

and its presence is associated with high mortality [45]. The mechanical ventilation in patients with ARDS can reduce the venous return due to the positive intrathoracic pressure and increase the RV afterload by lung volume changes which can markedly alter the pulmonary vascular resistance (PVR) [45]. The increased PVR leads to RV-pulmonary arterial
(PA) uncoupling with subsequent development and/or progression of RV dysfunction [37]. These side effects of ventilation can be exacerbated by the use of high positive endexpiratory pressure (PEEP) and were observed particularly often in CARDS accompanied by pulmonary small vessel thrombosis $[15,46]$. This also explains why decreased PEEP 
Fig. 1 Pathophysiologic role of heart-lung interactions for the development and progression of life-threatening RV dysfunction during SARS-COV-2 infection. The bold red arrows indicate the major direct impact of the virus on the lung tissue. The red dotted arrows indicate other possible direct cardiovascular damages of the virus with prognostic relevance

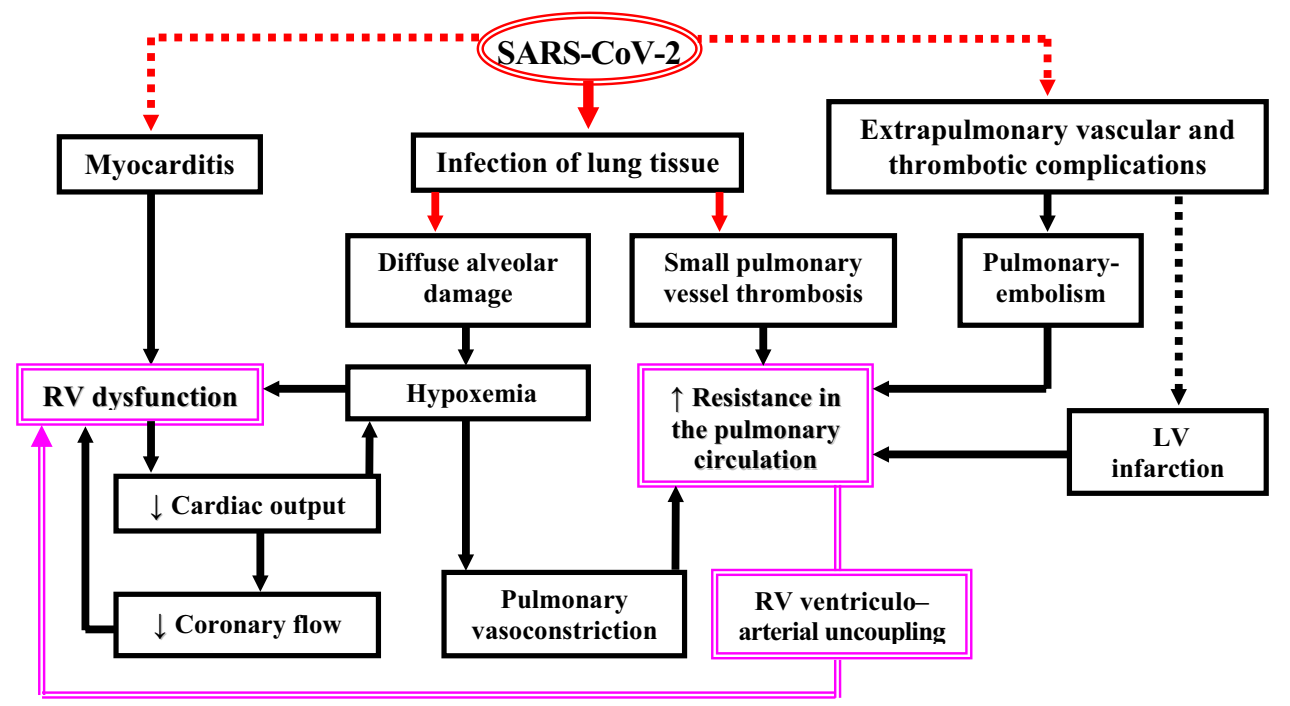

appeared independently associated with improved survival of patients with severe COVID-19 [15].

RV dilation and dysfunction also affect the LV loading and ejection by ventricular interdependence. The resulting reduction of the cardiac output, which impairs the blood supply to vital organs, can further aggravate the critical condition of the patient by exposing the overloaded RV to ischemia. In CARDS all this is amplified by the pulmonary microangiopathy with small vessel thrombosis which explains the particularly high incidence of cardiorespiratory failure in critically ill COVID-19 patients [2, 19].

A possible cause of COVID-19-related cardiac dysfunction is also the development of myocarditis which is a recognized severe complication of the virus infection [2]. The exact pathophysiology of SARS-CoV-2-associated myocarditis remains still not clearly definable. Direct virus-induced myocardial injury is possible since cardiomyocytes express a high level of ACE2 [47]. However, isolation of the virus from myocardial tissue was reported only in few autopsy studies and pathological reports have also described inflammatory infiltrates without myocardial evidence of SARSCoV-2 [20, 48]. Virus-host interactions with participation of both innate and acquired immune responses, as well as autoimmune mediated mechanisms also contribute to SARS-CoV-2 myocardial injury. In a clinical study without availability of myocardial biopsies, although $12.5 \%$ of the investigated COVID-19 patients had cardiac abnormalities that could indicate myocarditis, the changes of cardiac hsTnI over time and the absence of typical signs on ECG and TTE have suggested that myocardial injury might have been more likely related to indirect deleterious effects resulting from hyperactivation of inflammation and/or consequences of altered heart-lung interaction rather than direct virusmediated cell damage [49]. Thus, although signs of infection can be detected in myocardial tissues, cardiac involvement in COVID-19 is possibly more integrated with systemic disorders. This supports the current recommendation to be rather reluctant to make use of cardiac biopsy for COVID19 patients with suspected myocarditis because of the high risks of spreading the virus.

\section{Impact of ventilatory support on heart-lung interactions}

Although the entrance portal for SARS-CoV-2 is inhalational, and alveolar infiltrates are commonly detectable, the CARDS usually includes a major pulmonary vascular insult (endothelial injury with highly activated coagulation cascade) with particularly severe impairment of heart-lung interaction that may also require specific treatment approaches than usually applied for ARDS. The large disparities in mortality rates across different ICUs may also indicate that the approach to ventilatory management could indeed affect patient outcome [50].

The hemodynamic effects of mechanical ventilation (MV) are mainly induced by changes in pleural pressure and transpulmonary pressure (TP). MV can reduce the venous return due to the positive intra-thoracic pressure and increase the RV afterload by increasing the TP [46]. Tidal forces and PEEP increase PVR in direct proportion to their effects on mean airway pressure [46]. The particularly recommended lung protective ventilation strategies in CARDS refer mainly to PEEP, which, by modifying both stress and strain, contributes to the applied mechanical power [50,51]. Whereas optimal PEEP is beneficial by acting as a counterforce against superimposed pressure, thereby preventing atelectasis, and too low PEEP can induce atelectotrauma, high PEEP levels cause overinflation of the normal alveoli and compression of intraalveolar vessels, which lead to high PVR and 
thereby to increased RV afterload [43, 51]. RV dysfunction can therefore evolve as a deleterious consequence of MV. Being an early sign of RV overloading, RV dilatation during the course of MV requires particular attention.

COVID-19 causes quite unique lung injury, and the necessary respiratory support approaches depend largely on the underlying pathophysiology, which varies in accordance with the stage of disease. In the early stage of CARDS, although minute ventilation is characteristically high, many patients are not overtly dyspneic despite very poor oxygenation [50, 52]. In a simplified model, these patients are assigned to "type-L" disease characterized by low lung elastance (high compliance), lower lung weight (estimated by CT), low ventilation/perfusion mismatch, and low response to PEEP [50, $53,54]$. Due to their good lung compliance, these patients accept larger tidal volumes than those usually prescribed for ARDS, without increasing the risk of ventilator-induced lung injury (VILI) [50]. Wheareas for many patients, the disease may stabilize at this stage, some may progress to a more characteristic clinical picture of CARDS defined as "type-H," with extensive lung consolidations, low compliance, higher lung weight, high right-left shunt, and high PEEP response $[50,54]$. Between the 2 extremes, there are inter-mediate stages in which the type-L and type- $\mathrm{H}$ characteristics may overlap. Despite this over-lapping, it may be helpful to categorize patients as having either $\mathrm{L}$ or $\mathrm{H}$ phenotype. However, because of the severe widespread endothelial damage that promotes thrombogenesis in small pulmonary vessels and impairs the pulmonary vasoregulation (i.e., failure to develop a normal pulmonary vasoconstriction in response to hypoxia), the resulting ventilation-perfusion mismatch with life-threatening hypoxemia, becomes the key problem already in early CARDS stages [50].

Noninvasive respiratory support (e.g., high-flow nasal $\mathrm{O}_{2}$, continuous positive airway pressure [CPAP], noninvasive ventilation [NIV]) providing preconditions for the lowest possible mechanical load on the lungs may stabilize the course in mild cases $[50,54]$. The support must aim to minimize the volume and TP because high TP during inspiration and large tidal volumes will increase the lung stress and strain [50]. However, if the respiratory drive is not reduced by noninvasive support, the high drive and persistently strong spontaneous inspiratory efforts will result in patient "self-induced lung injury" [SILI] (mainly as a consequence of an excessive global and regional lung stress) followed by pulmonary transvascular pressure rise and fluid leakage with progressive deterioration of lung and RV function $[50,51,55]$. Timely intubation with effective sedation may interrupt this cycle and in patients with compliant lungs targeting higher tidal volumes and lower PEEP $(8-10 \mathrm{~cm}$ $\mathrm{H}_{2} \mathrm{O}$ ) will be appropriate [50]. If lung edema increases in type-L patients (because of the disease itself and/or SILI), the shrinking lung ("shrinking baby lung") shrinks further with a parallel decrease in respiratory system compliance, and converts progressively into a type-H phenotype [50, 51]. In this advanced state, it is advisable to apply a more conventional lung-protective strategy: $\mathrm{PEEP} \leq 15 \mathrm{~cm} \mathrm{H}_{2} \mathrm{O}$, lower tidal volume $(6 \mathrm{~mL} / \mathrm{kg})$, and prone positioning while minimizing $\mathrm{O}_{2}$ consumption [50]. Concentrating the entire ventilation workload on an already overstrained baby lung accentuates its potential for progressive lung injury. The forces of mechanical power (a function of strain, stress, and respiratory rate) are primarily applied to the extracellular matrix to which both the epithelial and endothelial cells are anchored [51]. The strain of these two cell populations contributes through a cytokine release to a further recruitment of inflammatory cells. This inflammatory reaction can severely alter the reaction to and tolerance of repeated cycles of high stress and strain [51]. Therefore, it can be necessary to consider also extractorporeal membrane oxygenation (ECMO) for some carefully selected patients with severe CARDS [50, 54, 56].

An important aspect related to both noninvasive and invasive $\mathrm{MV}$ is the negative impact of hyperoxia on the pulmonary microcirculation. This is of particular importance due to the fact that the anatomical and pathophysiological features of COVID-19-related lung infection are different from classic ARDS [57]. Exposure to hyperoxia over several days/weeks can induce complex endothelial, epithelial, platelet, and immune cell responses in the lung resulting in diffuse alveolar damage [58]. Given the spatial heterogeneity of pulmonary involvement in COVID-19, it is likely that segments of the severely damaged and largely consolidated lung experience lesser exposure to hyperoxia, whereas segments that are not consolidated and well ventilated are more prone to VILI and injury induced by hyperoxia. The vascular and immunological features of CARDS may reflect, at least in part, changes ascribed to prolonged hyperoxia that are superimposed on the original COVID-19-related disease, or develop in parallel in the well-ventilated segments of the lung [58]. There is evidence for overlapping pathological effects upon the lung of SARS-CoV-2 infection and exposure to high concentrations of inhaled $\mathrm{O}_{2}$ and hyperoxia may exacerbate the local cytopathic effects of the virus [58], therefore limiting the exposure to hyperoxia to the lowest levels that are required appeared advisable [58].

\section{Relevance of right heart dysfunction in COVID-19}

In a multicenter study, the most frequent ECHO abnormality in patients with COVID-19 was RV dilation with or without dysfunction [39]. In hospitalized patients, two different ECHO studies revealed RV dilation in $28 \%$ and $41 \%$, respectively, and RV dysfunction was evidenced in $27 \%$ of the patients in both studies $[59,60]$. In another study, RV dilation was present in $31 \%$ of the hospitalized COVID-19 
patients with similar prevalence of major comorbidities, laboratory markers of myocardial injury or inflammation, and also no differences in LV size and EF compared with patients without RV dilation [61]. Severely ill patients can develop isolated RVF, more often associated with increased PVR derived from PE and/or pulmonary small vessel thrombosis $[2,19]$. ECHO allowed the detection of elevated PAPs in up to 70\% of COVID-19 patients [59]. In hospitalized patients with COVID-19, ECHO revealed also a discrepancy between the involvements of the right-sided and left-sided heart in the prognosis of the disease [62]. Thus, non-survivors displayed significantly enlarged right heart chambers, reduced RV function, and elevated PAPs, whereas the LV and left atrial (LA) size as well as the LVEF were in the normal range [62]. Lower $\mathrm{RV}$ longitudinal strain (RVLS), $\mathrm{RV}$ fractional area change $\left(\mathrm{FAC}_{\mathrm{RV}}\right)$, and the lateral tricuspid annulus peak systolic excursion (TAPSE) as well as RV dilation were identified as powerful and independent predictors of higher mortality [61, 62]. In another study (100 COVID-19 patients), RV dilation and dysfunction were the most frequent ECHO abnormalities (detectable in 39 patients), whereas LVEF reduction occurred only in 8 patients [39]. Among the patients developing clinical deterioration during follow-up, $50 \%$ showed further deterioration of RV parameters, whereas the $\mathrm{LV}$ function remains normal in all those patients [39]. An ECHO study on hospitalized COVID-19 patients revealed RV dilation in $74 \%$ of them and the RV/LV ratio, which was increased in $82 \%$ of the patients, reached $0.9 \pm 0.3$ [63]. In that study, the LVEF was $60 \%$ (52-74\%) and reduction of LVEF was detected in only $3 \%$ of the patients. Heart transplanted patients with COVID19 , who needed mechanical ventilation, showed a mortality of $87.5 \%$, which appeared mainly associated with RV dysfunction (at simultaneously increased PAP), whereas none of them showed LVEF reduction [64]. The prevalence of $\mathrm{PH}$ (mean PAP $\geq 25 \mathrm{mmHg}$ ) was 4 times higher in ventilated COVID-19 patients with ARDS compared to those without the need for mechanical ventilation (76\% vs. 19\%) [65]. In another study, 29\% of COVID-19 patients treated in the ICU had PAP values $>40 \mathrm{mmHg}$ [19]. A previously normal RV (without pre-existing hypertrophy) will be barely able to generate such high pressures for several days, and therefore, such patients are at high risk to develop acute RV failure. In other studies, almost $50 \%$ of critically ill patients with COVID-19 revealed thrombotic events (including pulmonary microvascular occlusions) and about $22 \%$ experienced myocardial ischemia and/or AMI without signs of congestive HF [2]. In a study which included COVID-19 patients without previously known cardiomyopathy, ECHO parameters on LV function were not different between survivors and non-survivors [62].

Whereas, in general, LV failure is one of the most common etiologies of acute RV failure in the ICU, in critically ill COVID-19 patients, pulmonary tissue damages and small vessel thrombosis with consequent hypoxemia and excessive increase in the resistance in the pulmonary circulation are the cause of both acute respiratory and RV failure. Patients with cardiac injury (increased hs-TnI) experienced 3.6 times more $\mathrm{RV}$ than LV systolic dysfunction (47\% and $13 \%$, respectively) [66]. Timely identification of patients at high risk for RV failure can be life-saving for them. Although there are no specific biomarkers for RV failure, markers of myocardial injury like hs-TnI and N-terminal proBNP can help in prognostication because they have shown rapid increase in non-survivors. ECG lacks sensitivity and once chest X-ray or CT reveal RV dysfunction; RVF is usually advanced and associated with high mortality [67]. Pulmonary artery catheters (PACs) and ECHO are the most reliable tools to diagnose RV failure and evaluate the treatment response in the ICU. Their combined use provides crucial information for timely detection of potentially life-threatening alterations in the pulmonary circulation and RV function, as well as for ensuring the best possible patient care, including evaluation of the responses to therapies aimed at reducing RV afterload, optimizing the intravascular volume status and enhancing the RV inotropy. They can also help optimizing mechanical ventilation in order to avoid ventilator-induced lung injury and/or reduce its possible negative effects on RV preload and afterload $[9,68]$.

In specialized centers, ECHO is indispensable for the management of refractory respiratory failure with and without additional isolated RV failure or cardiogenic shock via artificial cardiopulmonary support strategies [56, 69-72]. Considering that without the option of ECMO therapy the mortality rate of patients with COVID-19-associated ARDS can reach $85 \%$ [2], the recently reported inhospital mortality of 38\% for ECMO-supported patients with CARDS [72], which is consistent with the mortality rates reported for nonCOVID-19 ECMO-supported patients, revealed the existence of important survival benefits achievable by ECMO therapy for appropriately selected patients with COVID-19. However, because in severe cases of RV afterload mismatch the RV output can decrease substantially even in the setting of normal intrinsic RV contractility (i.e., "acute corpulmonale"), in such situations, attempting to treat the underlying etiology of impaired gas exchange using V-V ECMO alone may not be sufficient because V-V ECMO recirculation is exacerbated by reduced RV ejection and TR [72]. Given the prognostic importance of the relatively high incidence of right heart involvement in COVID-19, close monitoring of $\mathrm{RV}$ size, geometry, and function in patients with refractory respiratory failure associated with hemodynamic instability, before overt RV failure develops, can substantially improve therapeutic decision-making [70]. ECHO can help with the selection of candidates for ECMO therapy, selection of the most appropriate support strategy (e.g., V-V ECMO plus catheter-mounted RV assist device [RVAD] or surgical 
RVAD plus gas exchanger), and is also necessary for weaning decision-making from the mechanical support, after cardio-pulmonary recovery $[56,69,70,72]$.

\section{Assessment of the right heart by echocardiography}

The frequently normal LVEF even in critically ill patients may have contributed to the initially restraint in use of ECHO for monitoring purposes in hospitalized patients with COVID-19. This is not surprising because for years, the assessment of the RV has lagged behind other ECHO examinations.

The main reasons for that were the historical lack of awareness of the pathophysiological and clinical importance of the RV function, the intense clinical focus of the left heart, and the paucity of normative data. More recently, RV function was found to be a powerful prognostic factor in $\mathrm{HF}$ and $\mathrm{PH}$ of various etiologies. Since mechanical circulatory support has become a well-established life-saving treatment for drug-refractory cardiorespiratory failure, assessment of RV size, geometry, and function has emerged as a crucial component of cardiac evaluation [69]. In patients with refractory respiratory failure associated with hemodynamic instability, TTE allows timely recognition of RV dysfunction (i.e., before overt RV failure develops) and could therefore spare some patients from additional ECMO therapy. Cardiologists already warned years ago that "it is imprudent to ignore the RV any longer " [73].

The increasing role of point-of-care (PoC) ECHO in the management of patients with undifferentiated shock in the emergency departments was also recently emphasized [74]. Given that pulmonary vessel injuries and blood flow alterations followed by RV failure are major causes of COVID-19-related deaths, it becomes increasingly difficult to follow the initial recommendation to avoid unnecessary cardiac imaging (including TTE) in order to reduce transmission of the virus and protect health care professionals because, without any ECHO examination, it cannot be established whether ECHO is indeed unnecessary in a given patient. Whereas the restraint use of trans-esophageal $\mathrm{ECHO}$ is fully justified by the high risk of the dispersion of droplets and aerosols, focused TTE protocols can provide a favorable benefit/risk balance in especially in patients with cardiovascular instability and/or suspicion of RV dysfunction $[75,76]$. With the ongoing COVID-19 pandemic, the increasingly recommended limited TTE with focused protocols, in particularly bedside PoC-TTE, can be indeed a good solution which considers both clinical and epidemiological concerns related to the management of hospitalized patients with COVID-19 [2, 76]. PoCUS, primarily focused on the pulmonary manifestations, can be particularly useful in the triage and decision making for COVID-19 patients [75, 76].
Compared with the $\mathrm{LV}$, the $\mathrm{RV}$ is less suited for quantitative ECHO-analysis, mainly because of its complex geometry with a limited number of well-defined anatomical landmarks and its variable position in the thorax. Because of the relevant impact of LV size and function on RV geometry and performance, the complex and different contraction-relaxation mechanism among the main segments of the RV, and the particularly high dependency of RV size, geometry, and function on RV loading conditions, even in the presence of unaltered myocardial contractility, collection of ECHO-data and especially their accurate interpretation is often a very challenging task $[73,77]$.

\section{Main objectives of right heart ECHO in severe COVID-19}

Detection and monitoring of right-sided heart dilation, RV dysfunction, tricuspid regurgitation (TR), and alterations of inferior vena cava size and blood flow are major goals of ECHO examinations in critically ill COVID-19 patients. In patients with RV dysfunction, it is also important to establish whether dysfunction is induced by pressure overloading or by impaired contractility or even by both.

\section{ECHO-assessment of right heart anatomy}

The apical 4-chamber (A4C) view is critical for assessment of right heart size and geometry, but the measurements rely on good visualization of the RV free wall and lateral tricuspid annulus. If apical images are poor, subcostal windows may provide similar views of the RV, but the difficulties in defining the anteroposterior plane of the RV from this window can induce misinterpretations in the evaluation of RV size [78]. Parasternal views allow measurements of RV outflow tract (RVOT) dimensions but the RVOT should be measured in both parasternal long-axis and short-axis views, in order to avoid measuring errors generated by patient position during the acquisition of RVOT images or rotation of the RVOT out of the section plane [77, 78]. During a limited bedside TTE, an A4C view is usually sufficient for assessment of right heart anatomy (including the TA) and the relationship between the size of right and left heart cavities, as well as for assessment of IVS and IAS position.

\section{ECHO-assessment of right ventricular function}

Assessment of RV function is more challenging than the assessment of its anatomical characteristics, and the interpretation of functional parameters for the $\mathrm{RV}$ is more demanding than for the LV [70]. RV pump performance depends mainly on myocardial contractility and loading conditions (preload and afterload) and is also influenced by heart rhythm, valve function, ventricular interdependence, and synchrony of ventricular contraction $[73,74]$. 
Mechanical and timing heterogeneity even in normal RVs make the assessment of systolic function particularly challenging. For objective reasons (e.g., complex RV shape necessitating geometric assumptions for volume calculations, limited imaging window, limited definition of end-systolic RV endocardial surface), RVEF calculations from 2D-ECHO-derived measurements are less reliable and therefore not anymore recommended for clinical use $[78,79]$. Nevertheless, 2D-ECHO is useful for assessment of RV function because it allows easy measurements of $\mathrm{FAC}_{\mathrm{RV}}$ and TAPSE, which provide similar information to RVEF [80-82]. TAPSE and $\mathrm{FAC}_{\mathrm{RV}}$ require no geometric assumptions, and TAPSE can be obtained even with poor image quality [77, 78]. During a limited bedside TTE, an $\mathrm{A} 4 \mathrm{C}$ view (preferably RV-focused view) is sufficient also for assessment of RV systolic function because both $F_{A C}$ and TAPSE are measured in this view. An additional subcostal view can provide useful data for assessment of TR.

There is evidence that severe COVID-19 ARDS can be associated with a specific phenotype of RV radial impairment with sparing of longitudinal function and TAPSE may become therefore less useful for detection of RV dysfunction [78]. In one study, although reduced $F_{A C} C_{R V}$ was present in about $70 \%$ of critically ill COVID-19 patients, TAPSE remained normal in nearly $80 \%$ of these patients [83]. However, in another study, patients with COVID-19-induced ARDS, those who survived, had significantly higher TAPSE values $(p<0.001)$ compared to those who died in the ICU [16]. A limitation of RVEF, $F_{A C}$, and TAPSE is the fact that due to their load dependency, they can change without changes in RV myocardial contractility. Thus, they will decrease with increasing PVR, even if myocardial contractility remains unaltered [81, 84, 85]. Additionally, TR can induce RVEF, $F_{A C}$, and TAPSE changes which can be misleading for assessment of RV contractile function and estimation of RV myocardial contractility [77-79, 81, 85-87]. TR can induce overestimations of RV contractile function by facilitating the RV free wall transversal (inward) and longitudinal motion which will increase the measured TAPSE, $\mathrm{FAC}_{\mathrm{RV}}$, and $\mathrm{EF}$, correspondingly to the increased blood volume leaving the RV in systole [85].

Alongside with the $\mathrm{FAC}_{\mathrm{RV}}$, also the RVOT velocity-time integral $\left(\mathrm{RVOT}_{\mathrm{VTI}}\right)$ can be useful for detection of RV dysfunction in hospitalized patients with COVID-19 [83]. Thus, reduced RVOT $_{\mathrm{VTI}}$ (i.e., $<19 \mathrm{~cm}$ ) was present in about $85 \%$ of critically ill COVID-19 patients, whereas the RV free wall strain was reduced in only about $30 \%$ of the patients [83].

The major challenge in assessing the RV involvement in the prognosis of COVID-19 is the appropriate incorporation of ECHO measurements into an integrative evaluation of the right-sided heart in connection with the RV myocardial contractile abilities and the impact of heart-lung interactions on RV size, geometry, and pump performance [88]. There is no individual ECHO-parameter, irrespective of whether derived from conventional ECHO, 3D-ECHO, tissue Doppler, or deformation (strain and strain rate) imaging, which can alone enable the distinction between high afterloadinduced and impaired contractility-induced RV dysfunction (Table 2).

\section{Distinction between right ventricular dysfunction with and without impaired myocardial contractility}

A crucial question which arises particularly in patients with severe COVID-19 is whether RV dysfunction is induced by pressure overloading (afterload mismatch) or by impaired contractility, or by both.

Assessment of RV-PA coupling, using the $\mathrm{FAC}_{\mathrm{RV}} / \mathrm{RV}$ systolic pressure (RVSP) and TAPSE/RVSP as indices of $\mathrm{RV}$ contractile function and prognosis [89], could therefore be useful in assessing and monitoring of RV function in patients with COVID-19 [16]. Using a standard cut off for normal function of 1.0, $\mathrm{FAC}_{\mathrm{RV}} / \mathrm{RVSP}$ identified $86 \%$ of hospitalized patients with COVID-19 as having RV-PA uncoupling [83]. Significant uncoupling $\left(\mathrm{FAC}_{\mathrm{RV}} / \mathrm{RVSP}<0.6\right)$ was detectable in $50 \%$ of ICU-treated patients with COVID-19 [83]. In patients with COVID-induced ARDS, the mortality rate within the first 20 days was twice as high in those with a TAPSE/PASP ratio $<0.635$, and reduction of TAPSE/PASP was identified as an independent predictor of mortality [16].

RV myocardial contractile abilities are also reflected by the relationship between RV pressure load and alteration of its geometry and size. Integrative approaches using parameter combinations which consider also the RV afterload, appeared particularly useful for evaluation of RV function $[85,90,91]$.

An easy approach to assess this relationship by ECHO is the calculation of the "RV load-adaptation index" $\left(\mathrm{LAI}_{\mathrm{RV}}\right)$, a composite variable based on the relationship between RV load and cavity dilation, also taking the RA pressure into account (i.e., ratio between the systolic mean $\Delta \mathrm{P}_{\mathrm{RV}-\mathrm{RA}}$ and $\mathrm{RV}$ end-diastolic volume per long-axis length) [85, 91]. Using the TR velocity-time integral $\left(\mathrm{VTI}_{\mathrm{TR}}\right)$ instead of $\Delta \mathrm{P}_{\mathrm{RV}-\mathrm{RA}}$ and the $\mathrm{RV}$ end-diastolic area $\left(\mathrm{A}_{\mathrm{ED}}\right)$ instead of the $\mathrm{RV}$ end-diastolic volume (EDV), the $\mathrm{LAI}_{\mathrm{RV}}$ defined accordingly as $\mathrm{LAI}_{\mathrm{RV}}=\mathrm{VTI}_{\mathrm{TR}} \bullet \mathrm{L}_{\mathrm{ED}} / \mathrm{A}_{\mathrm{ED}}$ becomes an easy calculable dimensionless index (Fig. 2) obtainable from a single ECHO view (i.e. A4C). A small RV area relative to long-axis length (unaltered size and geometry) in patients with high RV systolic pressure and relatively low RA pressure (i.e., high $\mathrm{VTI}_{\mathrm{TR}}$ ) yields a high $\mathrm{LAI}_{\mathrm{RV}}$ which indicates good adaptation to load (suggesting a good RV contractility) and the potential of the RV to improve its performance after reduction of loading conditions. A large $A_{\mathrm{ED}}$ relative to the long-axis length (spherical dilation) in patients with a relatively low $\mathrm{VTI}_{\mathrm{TR}}$ yields a low $\mathrm{LAI}_{\mathrm{RV}}$ indicating poor adaptation to load 


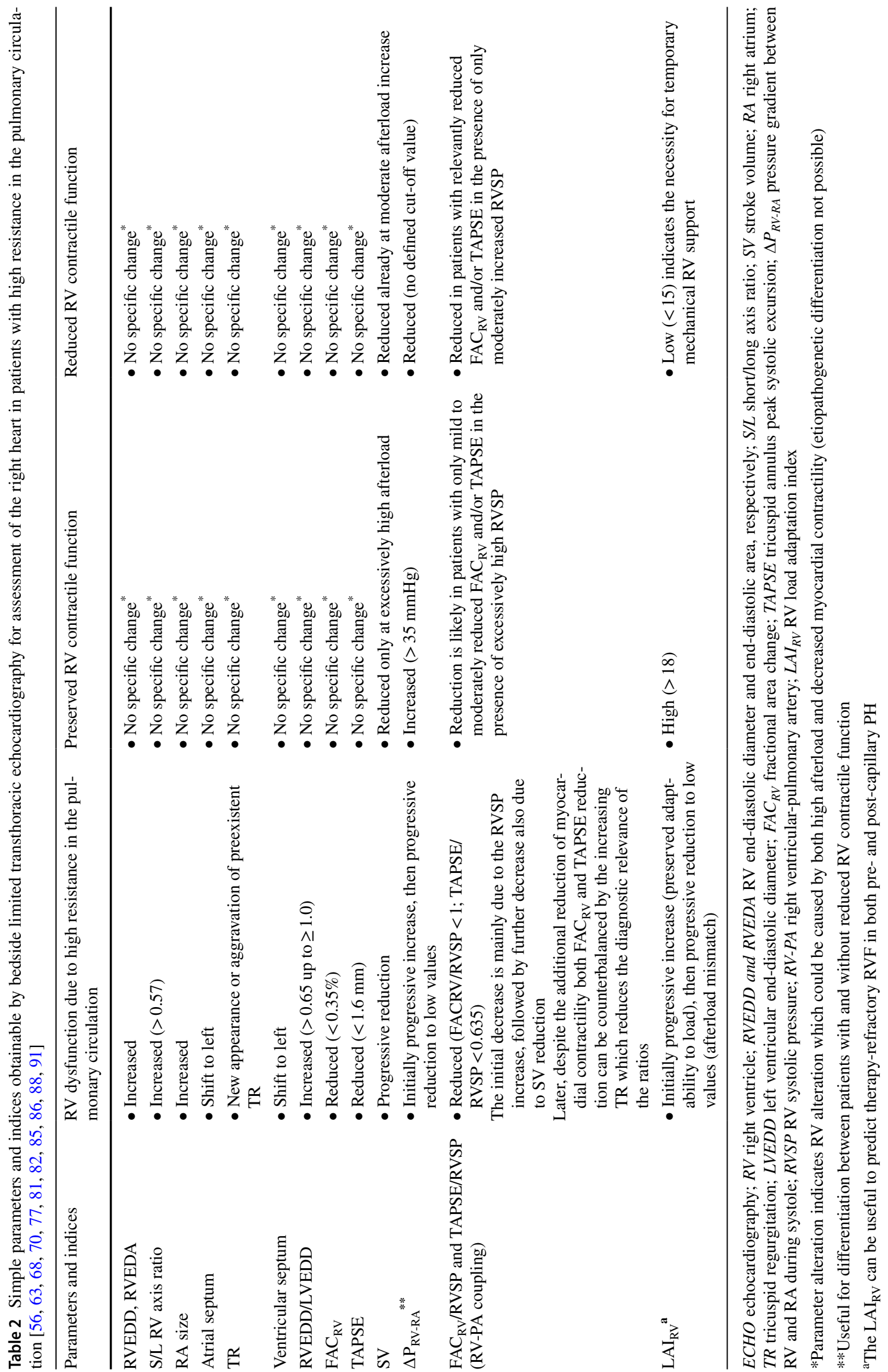




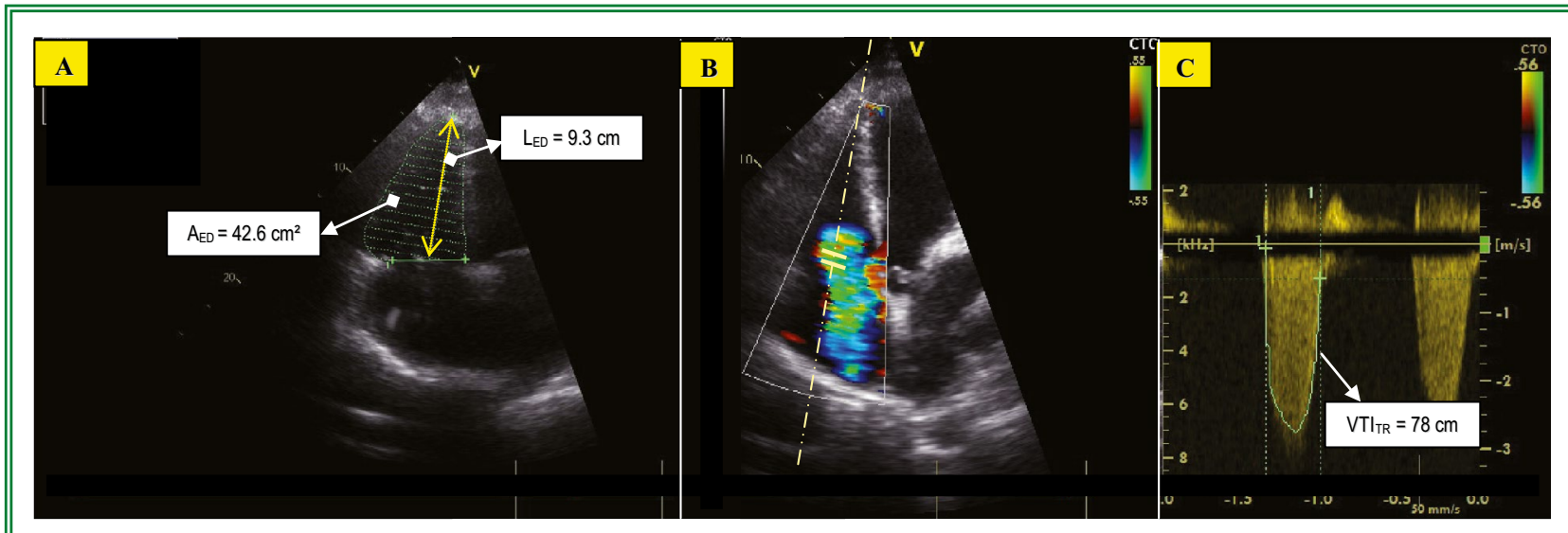

$$
\mathbf{L A I} I_{\mathrm{RV}}=\frac{\mathbf{V T I}_{\mathrm{TR}}(\mathrm{cm}) \cdot \mathbf{L}_{\mathrm{ED}}(\mathrm{cm})}{\mathbf{A}_{\mathrm{ED}}\left(\mathrm{cm}^{2}\right)}=\frac{\mathbf{7 8} \mathrm{cm} \cdot 9.3 \mathrm{~cm}}{42.6 \mathrm{~cm}^{2}}=\mathbf{1 7 . 0}
$$

Fig. 2 Calculation of the right ventricular load adaptation index $\left(\mathrm{LAI}_{\mathrm{RV}}\right)$. a Measurement of the right ventricular (RV) end-diastolic area $\left(A_{\mathrm{ED}}\right)$ and long axis lengths in the apical 4 chamber view. $\mathbf{b}$ and c Measurement of the tricuspid regurgitation velocity-time integral $\left(\mathrm{VTI}_{\mathrm{TR}}\right)$ using the continuous wave Doppler. The $\mathrm{LAI}_{\mathrm{RV}}$ value of 17.0 is reduced and indicates that in this patient with pulmonary

(suggesting a reduced $\mathrm{RV}$ myocardial contractility). $\mathrm{LAI}_{\mathrm{RV}}$ values $<15$ indicate severely impaired adaptability to load being usually insufficient to prevent RV failure even after normalization of PVR [77]. Good adaptability to high afterload does not exclude a reduced RV pump function which will occur during afterload mismatch. The $\mathrm{LAI}_{\mathrm{RV}}$ allows distinguishing between pressure overloading-induced and impaired contractility-induced RV dysfunction, as well as between patients with similar $\mathrm{FAC}_{\mathrm{RV}}$, but different RV myocardial inotropy [77]. In PAH, the $\mathrm{LAI}_{\mathrm{RV}}$ can reach values of 25 even in patients with relevant RV dysfunction, suggesting that in the vast majority of PAH patients, the cause of RV failure is the excessive pressure overload and not a relevantly impaired RV contractility [77, 85, 92]. This explains why RV failure is more likely and more faster reversible than LV failure of similar severity and also the reverse remodeling with normalization of RV function in the overwhelming majority of patients with pre-capillary PH-induced severe RV dysfunction, after they underwent lung transplantation [77, 85]. Therefore, temporary mechanical support of the RV can be taken into consideration in life-threatening COVID-19 [72, 93]. In such cases, differentiation between primary afterloadinduced and primary impaired contractility-induced RV failure can be crucial for a successful treatment.

\section{Bedside acquisition of essential ECHO data}

Considering the prognostic importance of right heart involvement in COVID-19, focusing more attention on the arterial hypertension (pulmonary arterial systolic and mean pressure: $48 \mathrm{mmHg}$ and $37 \mathrm{mmHg}$, respectively), the RV dilation is more pronounced than one would expect on the basis of its present afterload. The limited adaptation possibilities to the increased afterload are exceeded, and a reduction of pulmonary vascular resistance is highly needed in order to prevent further aggravation of RV dysfunction

RV during critical care ECHO can help in managing and monitoring patients with confirmed infection. Handheld laptop-based equipment is usually sufficient for the acquisition of essential ECHO data. An A4C view allows answering nearly all the major questions concerning the involvement of the right heart in COVID-19. An additional A5C view (easily acquirable from the $\mathrm{A} 4 \mathrm{C}$ ) plus a parasternal longaxis view enable the measurements necessary for calculation of the SV. In patients with relevant TR, a subcostal view can provide additional information for the severity of TR. Whereas the necessary TTE measurements are not time consuming, their interpretation remains more often the greater challenge. Although in most patients with severe COVID-19, TTE can provide the information needed, there are situations (e.g., patients with acute circulatory failure), in which critical care goal-directed TEE will become an invaluable diagnostic tool for managing such critically ill patients [94].

\section{Conclusions and further challenges}

Extensive pulmonary microangiopathy with small vessel thrombosis associated with particularly severe hypoxemic respiratory failure, high resistance in the pulmonary circulation, and life-threatening HF is a distinctive feature of COVID-19 and also a main cause for the particularly high mortality associated with the disease. Due to the direct alterations and damages of the pulmonary circulation, the pathophysiologic role of the heart-lung interactions becomes 
Fig. 3 Major signs of either right ventricular improvement or further deterioration revealed by echocardiographic monitoring of patients with increased resistance in the pulmonary circulation. RV right ventricle, TR tricuspid valve regurgitation, SV stroke volume, CO cardiac output, $\mathrm{LAI}_{\mathrm{RV}}$ right ventricular load adaptation index, TAPSE tricuspid annulus peak systolic excursion, $\mathrm{FAC}_{\mathrm{RV}} \mathrm{RV}$ fractional area change, RVLS right ventricular longitudinal strain

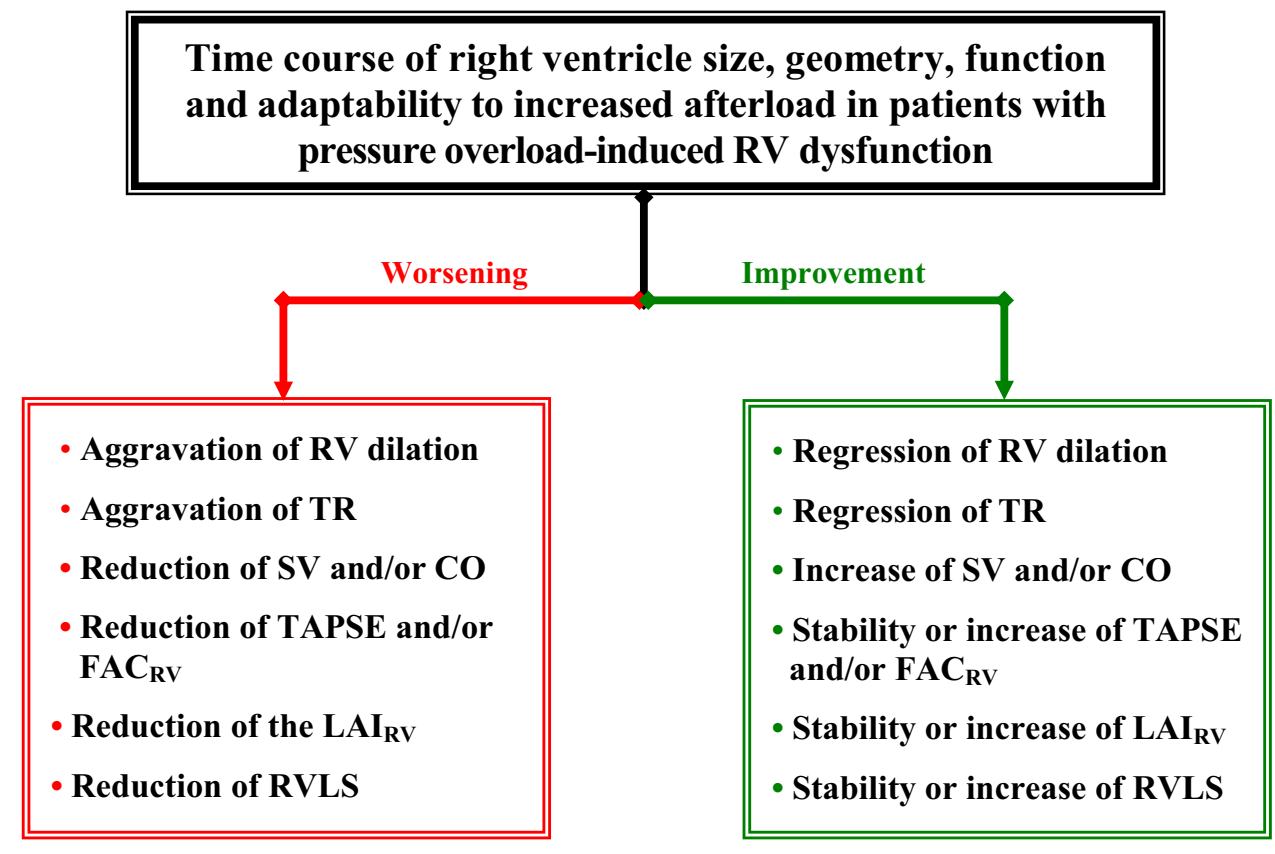

mainly involved in the development and progression of severe RV dysfunction. By contrast, severe reduction of LV pump function occurred more rarely (especially in patients without preexisting cardiovascular diseases). The close correlations between cardiac troponine and D-dimer levels with both PAPs and RV systolic dysfunction plus the correlation between D-dimer levels and RV dilation underline the pathogenetic significance of the COVID-19-associated pulmonary microvascular thrombosis-induced RV afterload increase. The high afterload-induced increase in RV wall tension associated with RV myocardial damage and dysfunction appeared to be the major cause for the COVID-19-related acute HF and troponin increase is mainly reflecting $\mathrm{RV}$ afterload increase.

Being the most impacted side of the heart in COVID19 and because RV dilatation and dysfunction were often identified as the most prominent ECHO abnormalities, the right heart deserves proper consideration. Along with biological markers like hs-TnI and D-dimers, ECHO can help anticipating a worsening of the patient's condition. Bedside limited TTE focused on the right heart is a useful diagnostic tool in hospitalized COVID-19 patients, particularly in those with suspicion of RV dysfunction or $\mathrm{PH}$ and becomes necessary for monitoring of critically ill patients. Even hand-held laptop-based equipment can be often sufficient for acquisition of the strictly necessary ECHO data and it appears advisable to further promote the routine use of this time- and cost-effective diagnostic tool in the management of symptomatic COVID-19 infected patients.

The particular pathophysiology of COVID-19 places the $\mathrm{RV}$ at higher risk of failure, and therefore, one key problem remains the timely recognition and severity grading of right heart involvement and the prediction of RV failure. In this regard, monitoring of RV and RA size, search for TR and assessment of its progress, as well as monitoring of the SV and the pressure gradient between the $\mathrm{RV}$ and $\mathrm{RA}\left(\Delta \mathrm{P}_{\mathrm{RV}-\mathrm{RA}}\right)$ are particularly useful (Fig. 3). Assessment of RV-PA coupling using the FAC/RVSP, plus evaluation of RV adaptability to pressure loading using the $\mathrm{LAI}_{\mathrm{RV}}$, can also allow prognostic statements to be made. Given the growing evidence of therapeutic successes achieved in highly selected COVID-19 patients with life-threatening refractory respiratory or cardiorespiratory failure using ECMO, whereas patients who are inappropriately selected stand a much lower chance of survival, the role of bedside TTE in identifying suitable patients and selection of the appropriate type of ECMO will also continue to grow.

Full implementation of bedside limited TTE focused on the right heart, which is currently still under-used, could facilitate more personalized management and treatment of hospitalized patients and can contribute towards reducing the high mortality associated with SARS- CoV-2 infection.

\section{Declarations}

Conflict of interest The authors declare no competing interests.

\section{References}

1. Wei Z-Y, Qian H-Y, Huang J, Yong-Jian Geng Y-J (2020) Pathogenesis and management of myocardial injury in Coronavirus disease. Eur J Heart Fail 22(11):1994-2006

2. Gupta A, Madhavan MV, Sehgal K et al (2020) Extrapulmonary manifestations of COVID-19. Nat Med 26(7):1017-1032 
3. Geng YJ, Wei ZY, Qian HY et al (2020) Pathophysiological characteristics and therapeutic approaches for pulmonary injury and cardiovascular complications of corona virus disease 2019. Cardiovasc Pathol 47:107228. https://doi.org/10.1016/j.carpath.2020. 107228

4. Clerkin KJ, Fried JA, Raikhelkar J et al (2020) COVID-19 and cardiovascular disease. Circulation 141(20):1648-1655

5. John Wort S, Arachchillage DJ, McCabe C, Price LC (2020) Covid-19 pneumonia and pulmonary vascular disease: A UK Centre perspective. Respir Med Res 78:100781. https://doi.org/ 10.1016/j.resmer.2020.100781 (Epub 2020 Aug 3)

6. Sakr Y, Giovini M, Leone M et al (2020) Pulmonary embolism in patients with coronavirus disease-2019 (COVID-19) pneumonia: a narrative review. Ann Intensive Care10:124. https://doi.org/10. 1186/s13613-020-00741-0. eCollection 2020

7. Huang C, Wang Y, Li X et al (2020) Clinical features of patients infected with 2019 novel coronavirus in Wuhan. China Lancet 395(10223):497-506

8. Papamanoli A, Yoo J, Grewal P (2020) High-dose methylprednisolone in nonintubated patients with severe COVID-19 pneumonia. Eur J Clin Invest 1:e13458

9. Goodacre S, Thomas B, Lee E et al (2020) Characterisation of 22445 patients attending UK emergency departments with suspected COVID-19 infection: Observational cohort study. PLoS ONE 15(11):e0240206

10. Lentz S, Roginski MA, Montrief T et al (2020) Initial emergency department mechanical ventilation strategies for COVID19 hypoxemic respiratory failure and ARDS. Am J Emerg Med 38(10):2194-2202

11. Shah SJ, Barisha PN, Prasada PA et al (2020) Clinical features, diagnostics, and outcomes of patients presenting with acute respiratory illness: A retrospective cohort study of patients with and without COVID-19. E Clin Med 100518. https://doi.org/10. 1016/j.eclinm.2020.100518

12. Donnelly JP, Wang XQ, Iwashyna TJ, Prescott HC (2020) Readmission and death after initial hospital discharge among patients with COVID-19 in a large multihospital system. JAMA e2021465. https://doi.org/10.1001/jama.2020.21465. Online ahead of print

13. Giustino G, Croft LB, Stefanini GG et al (2020) Characterization of myocardial injury in patients with COVID-19. J Am Coll Cardiol 76(18):2043-2055

14. Zhou F, Yu T, Du R et al (2020) Clinical course and risk factors for mortality of adult inpatients with COVID-19 in Wuhan, China: a retrospective cohort study. Lancet 395:1054-1062

15. Grasselli G, Greco M, Zanella A et al (2020) Risk factors associated with mortality among patients with COVID-19 in intensive care units in Lombardy. Italy JAMA Intern Med 180(10): $1345-1355$

16. D'Alto M, Marra AM, Severino S et al (2020) Right ventricular-arterial uncoupling independently predicts survival in COVID-19 ARDS. Crit Care 24(1):670. https://doi.org/10.1186/ s13054-020-03385-5

17. Màrca $\mathrm{J}$, Jor $\mathrm{O}$, Holub $\mathrm{M}$ et al (2017) Past and present ARDS mortality rates: a systematic review. Respir Care 62(1):113-122

18. Long B, Bradly WJ, Koyfman A, Gottlieb M (2020) Cardiovascular complications in COVID-19. Am J Emerg Med 38(7):1504-1507

19. Zeng J-H, Wu W-B, Qu J-X et al (2020) Cardiac manifestations of COVID-19 in Shenzhen. China Infection 48(6):861-870

20. Chen W, Pan JY (2021) Anatomical and pathological observation and analysis of SARS and COVID-19: microthrombosis is the main cause of death. Biol Proced Online 23(1):4. https://doi.org/ 10.1186/s12575-021-00142-y

21. Bösmüller H, Traxler S, Bitzer M et al (2020) The evolution of pulmonary pathology in fatal COVID-19 disease: an autopsy study with clinical correlation. Virchows Arch 477(3):349-357
22. Fox SE, Akmatbekov A, Harbert JL et al (2020) Pulmonary and cardiac pathology in African American patients with COVID19: an autopsy series from New Orleans. Lancet Respir Med 8:681-686

23. Ackermann M, Verleden SE, Kuehnel M et al (2020) Pulmonary vascular endothelialitis, thrombosis, and angiogenesis in Covid19. N Engl J Med 383(2):120-128

24. Dolhnikoff M, Duarte-Neto AN, de Almeida Monteiro RA et al (2020) Pathological evidence of pulmonary thrombotic phenomena in severe COVID-19. J Thromb Haemost 18:1517-1519

25. Carsana L, Sonzogni A, Nasr A et al (2020) Pulmonary post-mortem findings in a series of COVID-19 cases from northern Italy: a twocentre descriptive study. Lancet Infect Dis 20:1135-1140

26. Wichmann D, Sperhake J-P, Lütgehetmann M et al (2020) Autopsy findings and venous thromboembolism in patients with COVID-19. Ann Intern Med 173(4):268-277

27. Gallastegui N, Zhou JZ, Drygalski AV et al (2021) Pulmonary embolism does not have an un-usually high incidence among hospitalized COVID19 patients. Clin Appl Thromb Hemost 27. https://doi.org/10.1177/1076029621996471

28. Scudiero F, Silverio F, Di Maio M et al (2021) Pulmonary embolism in COVID-19 patients: prevalence, predictors and clinical outcome. Thromb Res 198:34-39

29. Sheng L, Wang X, Tang N et al (2021) Clinical characteristics of moderate and severe cases with COVID-19 in Wuhan, China: a retrospective study. Clin Exp Med 21(1):35-39. https://doi.org/ 10.1007/s10238-020-00662-z

30. Grasselli G, Tonetti T, Protti A et al (2020) Pathophysiology of COVID-19-associated acute respiratory distress syndrome: a multicentre prospective observational study. Lancet Respir Med. Published Online August 27, 2020. https://doi.org/10.1016/S22132600(20)30370-2

31. Fox SE, Li G, Aakmatbekov A et al (2020) Unexpected features of cardiac pathology in COVID-19 infection. Circulation 142:1123-1125

32. Goudot G, Chorcron R, Augy JL et al (2020) Predictive factor for COVID-19 worsening: insights for high-sensitivity troponin and D-dimer and correlation with right ventricular afterload. Front Med (Lausanne) 7: 586307

33. Ruan Q, Yang K, Wang W et al (2020) Clinical predictors of mortality due to COVID-19 based on an analysis of data of 150 patients from Wuhan. China Intensive Care Med 46(5):846-848

34. Tomasoni D, Inciardi RM, Lombardi CM et al (2020) Impact of heart failure on the clinical course and outcomes of patients hospitalized for COVID-19. Results of the Cardio-COVID-Italy multicentre study. Eur J Heart Fail 22(12):2238-2247. https://doi. org/10.1002/ejhf.2052

35. Shi S, Qin M, Shen B et al (2020) Association of cardiac injury with mortality in hospitalized patients with COVID-19 in Wuhan. China JAMA Cardiol 5(7):802-810

36. Salvatici M, Barbieri B, Cioffi SMG et al (2020) Association between cardiac troponin I and mortality in patients with COVID19. Biomarkers 25(8):634-640

37. Guo T, Fan Y, Chen M et al (2020) Cardiovascular implications of fatal outcomes of patients with coronavirus disease 2019 (COVID-19). JAMA Cardiol 5(7):811-18

38. Giannitsis E, Müller-Bardorff M, Kurowski V et al (2000) Independent prognostic value of cardiac troponin $\mathrm{T}$ in patients with confirmed pulmonary embolism. Circulation 102:211-217

39. Szekely Y, Lichter Y, Taieb P et al (2020) Spectrum of cardiac manifestations in COVID-19: a systematic echocardiographic study. Circulation 142:342-353

40. Matsubara D, Hunter LK, Wang Y et al (2020) Echocardiographic findings in pediatric multi system inflammatory syndrome associated with COVID-19 in the United States. J Am Coll Cardiol 76(17):1947-1961. https://doi.org/10.1016/j.jacc.2020.08.056 
41. Thachil J, Tang N, Gando S et al (2020) ISTH interim guidance on recognition and management of coagulopathy in COVID-19. J Thromb Haemost 18(5):1023-1026

42. Bertini M, Ferrari R, Guardigli G et al (2020) Electrocardiographic features of 431 consecutive, critically ill COVID-19 patients: an insight into the mechanisms of cardiac involvement. Europace 22(12):1848-1854

43. Linschoten M, Peters S, van Smeden M et al (2020) Cardiac complications in patients hospitalized with COVID-19. Eur Heart J Acute Cardiovasc Care 9(8):817-823

44. Chen L, Li X, Chen M et al (2020) The ACE2 expression in human heart indicates new potential mechanism of heart injury among patients infected with SARS-CoV-2. Cardiovasc Res 116(6):1097-1100. https://doi.org/10.1093/cvr/cvaa078

45. Vieillard-Baron A, Naeije R, Haddad F et al (2018) Diagnostic workup, etiologies and management of acute right ventricle failure: a state-of-the-art paper. Intensive Care Med 44:774-790

46. Tsolaki V, Siempos I, Magira E et al (2020) PEEP levels in COVID-19 pneumonia. Crit Care 24:303. https://doi.org/10.1186/ s13054-020-03049-4

47. Magadum A, Kishore R (2020) Cardiovascular Manifestations of COVID-19 Infection. Cells 9(11):2508. https://doi.org/10.3390/ cells 9112508

48. Tavazzi G, Pellegrini C, Maurelli M et al (2020) Myocardial localization of coronavirus in COVID-19 cardiogenic shock. Eur J Heart Fail 22(5):911-915

49. Deng Q, Hu B, Zhang Y, Wang H et al (2020) Suspected myocardial injury in patients with COVID-19: Evidence from front-line clinical observation in Wuhan. China Int J Cardiol 311:116-121

50. Marini JJ, Gattinoni L (2020) Management of COVID-19 respiratory distress. JAMA 323(22):2329-2330

51. Gattinoni L, Meissner K, Marini JJ (2020) The baby lung and the COVID-19 era. Intensive Care Med 46(7):1438-1440

52. Grasselli G, Zangrillo A, Zanella A et al (2020) Baseline characteristics and outcomes of 1591 patients infected with SARSCoV-2 admitted to ICUs of the Lombardy Region. Italy JAMA 323(16):1574-1581

53. Gattinoni L, Chiumello D, Caironi P et al (2020) COVID-19 pneumonia: different respiratory treatments for different phenotypes? Intensive Care Med 46(6):1099-1102

54. Esnault P, Cardinale M, Hraiech S et al (2020) High respiratory drive and excessive respiratory efforts predict relapse of respiratory failure in critically ill patients with COVID-19. Am J Respir Crit Care Med 202(8):1173-1178

55. Pfeifer M, Ewig S, Voshaare T et al (2020) Position paper for the state-of-the-art application of respiratory support in patients with COVID-19. Respiration 99(6):521-542. https://doi.org/10.1159/ 000509104 (Epub 2020 Jun 19)

56. Pham DT, Toeg H, De Paulis R, Atluri P (2020) Establishment and management of mechanical circulatory support during the COVID-19 pandemic. Circulation 142(1):10-13

57. Dobler CC, Murad MH, Wilson ME (2020) Noninvasive positive pressure ventilation in patients with COVID-19. Mayo Clin Proc 95(12):2594-2601

58. Hanidziar D, Robson SC (2021) Hyperoxia and modulation of pulmonary vascular and immune responses in COVID-19. Am J Physiol Lung Cell Mol Physiol 320: L12-L16

59. García-Cruz E, Manzur-Sandoval D, Rascón-Sabido R et al (2020) Critical care ultrasonography during COVID-19 pandemic: the ORACLE protocol. Echocardiography. https://doi.org/10.1111/echo.14837

60. Mahmoud-Elsayed HM, Moody WE, Bradlow WM (2020) Echocardiographic findings in patients with COVID-19 pneumonia. Can J Cardiol 36(8):1203-1207

61. Argulian E, Sud K, Vogel B et al (2020) Right ventricular dilation in hospitalized patients with COVID-19 infection. JACC Cardiovasc Imaging 13(11):2459-2461
62. Li Y, Li H, Zhu S (2020) Prognostic value of right ventricular longitudinal strain in patients with COVID-19. J Am Coll Cardiol Img 13(11):2287-2299

63. Schott JP, Mertens AN, Bloomingdale R et al (2020) Transthoracic echocardiographic findings in patients admitted with SARSCoV-2 infection. Echocardiography 37(10):1551-1556

64. Rivinius R, Kaya Z, Schramm R et al (2020) COVID-19 among heart transplant recipients in Germany: a multicenter survey. Clin Res Cardiol 109(12):1531-1539

65. Caravita S, Baratto C, Di Marco F et al (2020) Haemodynamic characteristics of COVID-19 patients with acute respiratory distress syndrome requiring mechanical ventilation. An invasive assessment using right heart catheterization. Eur J Heart Fail 22(12):2228-2237. https://doi.org/10.1002/ejhf.2058

66. Doyen D, Dupland P, Morand L et al (2020) Characteristics of cardiac injury in critically ill patients with coronavirus disease 2019. Chest S0012-3692(20)35109-6. https://doi.org/10.1016/j. chest.2020.10.056. Online ahead of print

67. Rozental O, Thalappillil R, White R et al (2020) Hemodynamic monitoring options in COVID-19. J Cardiothorac Vasc Anesth 34(12):3488-3490

68. Cameli M, Pastore MC, Aboumarie HS et al (2020) Usefulness of echocardiography to detect cardiac involvement in COVID-19 patients. Echocardiography 37(8):1278-1286

69. Dandel M, Hetzer R (2018) Temporary assist device support for the right ventricle: pre-implant and post-implant challenges. Heart Fail Rev 23(2):157-171

70. Dandel M, Potapov E, Moazami N (2017) Preoperative evaluation of right ventricular function. In: A. Montalto, A. Loforte, F. Musumeci, T. Krabatsch and MS Slaughter (eds.) Mechanical circulatory support in end-stage heart failure. Springer Nature, Switzerland. p.76-90

71. Rajagopal K, Keller SP, Akkanti B et al (2020) Advanced pulmonary and cardiac support of COVID-19 patients: Emerging recommendations from ASAIO — a "Living Working Document." ASAIO J 66(6):588-598

72. Barbaro RP, MacLaren G, Boonstra PS et al (2020) Extracorporeal membrane oxygenation support in COVID-19: an international cohort study of the Extracorporeal Life Support Organization registry. Lancet 396(10257):1071-1078. https://doi.org/10.1016/ S0140-6736(20)32008-0

73. Walker LA, Buttrick PM (2009) The right ventricle: biologic insights and response to disease. Curr Cardiol Rev 5(1):22-28

74. Chenkin J, Atzema CL (2018) Contemporary Application of point-of-care echocardiography in the emergency department. Can J Cardiol 34(2):109-116

75. Skulstad H, Cosyns B, Popescu BA et al (2020) COVID-19 pandemic and cardiac imaging: EACVI recommendations on precautions, indications, prioritization, and protection for patients and healthcare personnel. Eur Heart J Cardiovasc Imaging 21(6):592-598

76. Dandel M. Pathophysiology of COVID-19-associated acute respiratory distress syndrome. Lancet Respir Med 2021; Jan;9(1):e4

77. Dandel M, Hetzer R (2018) Evaluation of the right ventricle by echocardiography: particularities and major challenges. Expert Reviews of Cardiovascular Therapy 16(4):259-275

78. Kurtz CE (2012) Right ventricular anatomy, function, and echocardiography evaluation. In Catherine Otto; The Clinical Practice of Echocardiography; $4^{\text {th }}$ edition $615-28$

79. Rudski LG, Lai WW, Afilalo J et al (2010) Guidelines for echocardiographic assessment of right heart in adults: A report of the American Society of Echocardiography. J Am Soc Echocardiogr 23:685-713

80. Guazzi M (2020) TAPSE/PASP use in heart failure: Implementing the evidence for simplicity Monaldi Arch Chest Dis 90(3). https:// doi.org/10.4081/monaldi.2020.1363 
81. Dandel M (2016) Hetzer R. Echocardiographic assessment of the right ventricle: Impact of the distinctly load dependency of its size, geometry and function. Int J Cardiol 221:1132-1142

82. Anavekar NS, Gerson D, Skali H et al (2007) Two-dimensional assessment of right ventricular function. An echocardiographicMRI correlative study. Echocardiography 24:452-456

83. Bleakley C, Singh S, Garfield B et al (2021) Right ventricular dysfunction in critically ill COVID-19 ARDS. Int J Cardiol 327:251-58. https://doi.org/10.1016/j.ijcard.2020.11.043

84. Dandel M, Hetzer R (2019) Echocardiographic assessment of the right-sided heart for surveillance of patients with pulmonary arterial hypertension. JACC Cardiovasc Imaging 12(4):764-766

85. Dandel M, Knosalla C, Kemper D et al (2015) Assessment of right ventricular adaptability to loading conditions can improve the timing of listing to transplantation in patients with pulmonary arterial hypertension. J Heart Lung Transplant 34(3):319-328

86. Hsiao SH, Lin SK, Wang WC (2006) Severe tricuspid regurgitation shows significant impact in the relationship among peak systolic tricuspid annular velocity, tricuspid annular plane systolic excursion, and right ventricular ejection fraction. J Am Soc Echocardiogr 19:902-910

87. Dandel M (2020) Potential impact of tricuspid and mitral valve regurgitation on the diagnostic and prognostic value of ventricular ejection fraction. J Am Soc Echocardiogr 33(4):518

88. Fukui M, Cavalcante JL (2020) Relation among right ventricular dysfunction, lung damage, and mortality in patients with COVID19. JACC Cardiovasc Imaging 13(8):1858-1859
89. Guazzi M, Bandera F, Pelissero G et al (2013) Tricuspid annular plane systolic excursion and pul-monary arterial systolic pressure relationship in heart failure: an index of right ventricular contractile function and prognosis. Am J Physiol Heart Circ Physiol 305:H1373-H1381

90. Amsallem M, Aymami M, Hiesinger W et al (2019) Right ventricular load adaptability metrics in patients undergoing left ventricular assist device implantation. J Thorac Cardiovasc Surg 157(3):1023-33.e4

91. Dandel M, Potapov E, Krabatsch T et al (2013) Load dependency of right ventricular performance is a major factor to be considered in decision making prior to ventricular assist device implantation. Circulation 128:S14-S23

92. French S, Amsallem M, Ouazani N et al (2018) Non-invasive right ventricular load adaptability indices in patients with scleroderma-associated pulmonary arterial hypertension. Pulm Circ 8(3):2045894018788268

93. Renard LS, Fried JA, Abdally M et al (2020) Approach to acute cardiovascular complications in COVID-19 infection. Circ Heart Fail 13(7):e007220

94. Teran F, Burns KM, Narasimhan M et al (2020) Critical care transesophageal echocardiography in patients during the COVID19 pandemic. J Am Soc Echocardiogr 33:1040-47

Publisher's Note Springer Nature remains neutral with regard to jurisdictional claims in published maps and institutional affiliations. 